\title{
Holographic KMS relations at finite density
}

\author{
R. Loganayagam, ${ }^{a}$ Krishnendu Ray, ${ }^{b}$ Shivam K. Sharma ${ }^{a}$ and Akhil Sivakumar ${ }^{a}$ \\ ${ }^{a}$ International Centre for Theoretical Sciences (ICTS-TIFR), \\ Tata Institute of Fundamental Research, \\ Shivakote, Hesaraghatta, Bangalore 560089, India \\ ${ }^{b}$ Rudolf Peierls Centre for Theoretical Physics, University of Oxford, \\ Parks Road, Oxford, OX1 3PU, U.K. \\ E-mail: nayagam@icts.res.in, krishnendu.ray@physics.ox.ac.uk, \\ shivam.sharma@icts.res.in, akhil.sivakumar@icts.res.in
}

ABstRact: We extend the holographic Schwinger-Keldysh prescription introduced in [1] to charged black branes, with a view towards studying Hawking radiation in these backgrounds. Equivalently we study the real time fluctuations of the dual CFT held at finite temperature and finite chemical potential. We check our prescription using charged Dirac probe fields. We solve the Dirac equation in a boundary derivative expansion extending the results in [2]. The Schwinger-Keldysh correlators derived using this prescription automatically satisfy the appropriate KMS relations with Fermi-Dirac factors.

KEYwORDS: AdS-CFT Correspondence, Holography and condensed matter physics (AdS/CMT), Holography and quark-gluon plasmas, Quantum Dissipative Systems

ArXiv EPrint: 2011.08173 


\section{Contents}

1 Introduction 1

2 Hawking radiation in RN AdS backgrounds 2

3 The Dirac equation $\quad 6$

4 Gradient expansion $\quad 8$

5 Conclusions and future directions $\quad 9$

\section{Introduction}

Black holes radiate and their Hawking radiation closely mimic the fluctuations in a thermal bath. Interacting fields in black hole backgrounds can hence be used to model interacting thermal environments. There is however a technical obstacle to such an endeavour: one needs a practical formalism to compute interactions between ingoing quasi-normal modes and the outgoing Hawking fluctuations. Recently, a gravitational Schwinger-Keldysh (grSK) geometry $[3,4]$ has emerged as an arena where such computations can be performed with ease. This prescription is built on earlier work on real time holography [1, 5-16].

The Schwinger-Keldysh, or 'in-in', formalism [17, 18] is the most robust setting to study the real-time dynamics of non-equilibrium systems [19-23]. The central idea of the construction involves a path integral with every degree of freedom doubled, describing the evolution of a general non-equilibrium mixed state. For near-equilibrium mixed states, such a path integral can be computed by a dual gravitational saddle built by smoothly glueing two copies of the exterior of a black hole across their future horizons. This is the gravitational Schwinger-Keldysh (grSK) geometry alluded to above. While an ab initio derivation of this prescription is still not known, its validity and efficacy have been demonstrated in several instances [1-4].

In an accompanying work [2], a subset of the authors of this note show that for probe Dirac fermions, the grSK geometry reproduces the correct Fermi-Dirac statistical factors in real time correlations. This setup can be used to compute the influence phase of an external fermion probing the dual CFT at finite temperature. A natural generalisation of this work is to ask what happens if we consider near-equilibrium states with finite density/chemical potential. Such a generalisation is necessary to make contact with models of holographic condensed matter [24-33]. On the gravitational side, the relevant physics involves the RN-AdS black brane, its quasi-normal modes and its Hawking radiation.

In this note, we give a holographic prescription that computes real time correlators at finite chemical potential in the dual CFT. We do this via a geometry made by stitching two 
copies of RN-AdS black brane exteriors which we will term as the RN-SK saddle/geometry. that generalises grSK geometry. In section 2, we devise a method to write down the outgoing Hawking modes in this geometry when the ingoing modes are known. We test this method in section 3 using a probe Dirac field and show that it indeed yields the correct Fermi-Dirac physics (as well as the correct Kubo-Martin-Schwinger [34, 35] relations) at finite chemical potential. This is followed in section 4 by an explicit solution to Dirac equation upto second order in boundary derivative expansion and the corresponding influence functional. We conclude in section 5 with a summary and a discussion of future directions.

\section{Hawking radiation in $\mathrm{RN}$ AdS backgrounds}

In this section, we will describe how to construct Hawking modes in charged black branes when the ingoing quasi-normal modes are known. We will assume that the ingoing solution is naturally specified in ingoing Eddington-Finkelstein coordinates as an analytic function (possibly in a boundary derivative expansion as is the case, for example, in the fluid-gravity correspondence $[36,37])$. We will construct these Hawking modes using a bulk $\mathbb{Z}_{2}$ action which is dual to a $C P T$ transformation on the boundary CFT.

Let us begin with the Reissner-Nordström black brane solution in $\mathrm{AdS}_{d+1}$ written out in ingoing Eddington-Finkelstein time and a mock tortoise coordinate, $\zeta$ :

$$
\begin{aligned}
\mathrm{d} s^{2} & =-r^{2} f \mathrm{~d} v^{2}+i \beta r^{2} f \mathrm{~d} v \mathrm{~d} \zeta+r^{2} \mathrm{~d} \boldsymbol{x}_{d-1}^{2}, \\
\mathcal{A}_{M} \mathrm{~d} x^{M} & =\mathcal{A}_{v} \mathrm{~d} v=-\mu\left(\frac{r_{h}}{r}\right)^{d-2} \mathrm{~d} v .
\end{aligned}
$$

which is the solution of the Einstein-Maxwell bulk action, given by,

$$
\frac{1}{2 \kappa^{2}} \int \mathrm{d}^{d+1} x \sqrt{-g}\left[\mathcal{R}+d(d-1)-\frac{1}{g_{F}^{2}} \mathcal{F}_{M N} \mathcal{F}^{M N}\right],
$$

where $g_{F}$ is the gauge coupling constant and we have set the AdS radius to unity. Equivalently, we can replace the description in terms of the metric to one in terms of a set of orthonormal 1-forms [2, 38],

$$
E^{(v)}=\frac{r}{2} \mathrm{~d} v-\frac{f}{2} r(i \beta \mathrm{d} \zeta-\mathrm{d} v), \quad E^{(\zeta)}=\frac{r}{2} \mathrm{~d} v+\frac{f}{2} r(i \beta \mathrm{d} \zeta-\mathrm{d} v), \quad E^{(i)}=r \mathrm{~d} x^{i} .
$$

In the above equations, $f$ is the emblackening factor of the RN-AdS black brane, $\beta$ is its inverse Hawking temperature, $r_{h}$ its outer horizon radius, and $\mu$ its chemical potential. These quantities are, in turn, given in terms of the mass parameter $M$ and the charge parameter $Q$ of the black brane as,

$$
f(r) \equiv 1+\frac{Q^{2}}{r^{2 d-2}}-\frac{M}{r^{d}}, \quad \frac{1}{\beta} \equiv \frac{d r_{h}}{4 \pi}\left(1-\frac{(d-2) Q^{2}}{d r_{h}^{2 d-2}}\right), \quad \mu \equiv \frac{g_{F} Q}{r_{h}^{d-2}}\left[\frac{d-1}{2(d-2)}\right]^{\frac{1}{2}} .
$$

The ADM mass density $\mathcal{M}$ and the charge density $\mathcal{Q}$ are given by,

$$
\mathcal{M}=\frac{(d-1) M}{2 \kappa^{2}}, \quad \mathcal{Q}=\frac{\sqrt{2(d-1)(d-2)}}{g_{F} \kappa^{2}} Q .
$$




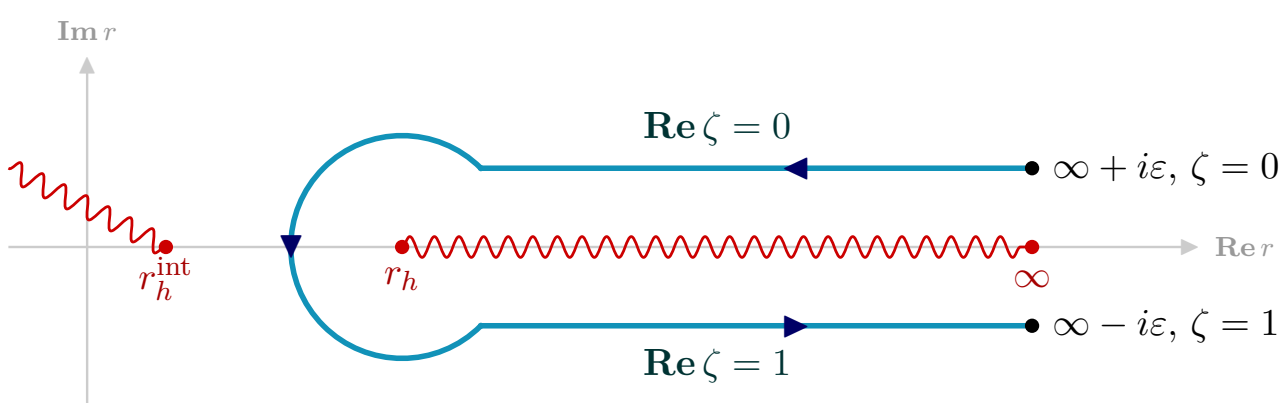

Figure 1. The radial contour drawn on the complex $r$ plane, at fixed $v$. The locations of the two boundaries and the two horizons have been indicated.

The mock tortoise coordinate $\zeta$ is related to the standard radial coordinate $r$ via the following differential equation,

$$
\frac{\mathrm{d} r}{\mathrm{~d} \zeta}=\frac{i \beta}{2} r^{2} f(r)
$$

Since the r.h.s. has simple zeroes at the inner and outer horizons, the coordinate $\zeta$, when thought of as a complex function on the complex $r$ plane, has these points (along with infinity and other complex simple zeroes of $f(r)$ ) as branch points. We will define $\zeta$ by taking a branch cut to extend from the outer horizon to the asymptotic boundary along the real axis. We choose the inner horizon branch cut such that $\zeta$ is analytic in the real interval between the two horizons (see figure 1). The differential equation above then normalises $\zeta$ such that it has a unit jump across the logarithmic branch cut. Without loss of generality, we will take $\zeta(r=\infty+i \varepsilon)=0$. This condition along with the above differential equation then uniquely defines $\zeta$ everywhere in the neighbourhood of $r \in\left[r_{h}, \infty\right)$.

We define the RN-SK geometry as one constructed by taking the RN-AdS exterior and replacing the radial interval extending from the outer horizon to infinity by a doubled contour, as indicated in figure 1. We then obtain a geometry with two copies of RN-AdS exteriors smoothly stitched together by a 'horizon cap' region. This spacetime requires one further identification - each radially constant slice must meet at the future turning point, $v \rightarrow \infty$.

Now that we have elaborated on the RN-SK geometry and its associated notation, let us return to the question posed at the beginning of this section: given an ingoing solution written in ingoing EF coordinates, how do we construct the outgoing Hawking modes? We will do this by exploiting the CPT symmetry of the boundary CFT which acts as,

$$
v \longmapsto-v, \quad \boldsymbol{x} \longmapsto-\boldsymbol{x},
$$

in addition to a charge conjugation. The reader can quickly verify that the transformations as written above do not preserve the RN-AdS solution given in (2.1) and (2.3). So how then do we implement this symmetry on the RN-AdS solution?

The answer to this conundrum lies in the fact that, by the standard rules of AdS/CFT, the boundary symmetries can always be composed with bulk gauge symmetries (since they act trivially on the Hilbert space of states of the CFT). In this case, we combine the 
boundary $C P T$ transformation with bulk diffeomorphisms, local Lorentz transformations and gauge transformations. In fact, the reader can check that the following transformations preserve the RN-AdS solution: we combine a charge conjugation $\mathcal{A}_{M} \mapsto-\mathcal{A}_{M}$, a diffeomorphism,

$$
v \longmapsto i \beta \zeta-v, \quad \boldsymbol{x} \longmapsto-\boldsymbol{x},
$$

a gauge transformation,

$$
\Lambda \equiv-i \beta \int_{0}^{\zeta} \mathrm{d} \zeta^{\prime} \mathcal{A}_{v}\left(\zeta^{\prime}\right)
$$

and a non-orthochronous local Lorentz transformation,

$$
\mathcal{T}^{a}{ }_{b} \equiv\left(\begin{array}{ccc}
-1 & 0 & 0 \\
0 & 1 & 0 \\
0 & 0 & -\mathbb{1}
\end{array}\right)\left(\begin{array}{ccc}
\cosh \vartheta & \sinh \vartheta & 0 \\
\sinh \vartheta & \cosh \vartheta & 0 \\
0 & 0 & \mathbb{1}
\end{array}\right), \quad \vartheta \equiv \log f .
$$

The linear transformation $\mathcal{T}$ is an idempotent local Lorentz transformation (LLT) composed of a boost along $\zeta$ with rapidity $\vartheta$ followed by a reflection of the $v$ and $\boldsymbol{x}$ axes.

To check the invariance of the geometry, we will write the diffeomorphism in eq. (2.8) in the form $X^{A} \mapsto X^{B} \mathcal{J}_{B}{ }^{A}$ where,

$$
\mathcal{J}_{A}{ }^{B} \equiv\left(\begin{array}{ccc}
-1 & 0 & 0 \\
i \beta & 1 & 0 \\
0 & 0 & -\mathbb{1}
\end{array}\right)
$$

The invariance is then the statement that

$$
\mathcal{T}^{a}{ }_{b} \mathcal{J}_{A}{ }^{B} E_{B}^{b}=E_{A}^{a}, \quad-\left(\mathcal{J}_{N}{ }^{M} \mathcal{A}_{M}+\partial_{N} \AA\right)=\mathcal{A}_{N},
$$

as can be readily checked.

It is instructive to unify these transformations by imagining the gauge field as descending from a Kaluza-Klein reduction over a circle. Say there was an extra circle direction denoted by $\varphi$, along which we have a 1 -form, $\mathrm{d} \varphi+\mathcal{A}_{N} \mathrm{~d} X^{N}$, that is orthonormal to the set of 1-forms, $E_{M}^{a} \mathrm{~d} X^{M}$. The action of charge conjugation and gauge transformation in this picture can then be thought of as the KK diffeomorphism $\varphi \mapsto \wedge-\varphi$ (which extends $\mathcal{J}_{A}{ }^{B}$ to the $\varphi$ direction) along with a KK LLT that acts as a reflection along the $\varphi$ axis (which extends $\mathcal{T}_{b}^{a}$ to the $\varphi$ direction). The mathematically inclined reader would recognise that we have essentially given a description of the boundary CPT transformation as an automorphism of the RN-AdS principle bundle.

Having implemented the boundary $C P T$ in the bulk, we will now use this symmetry to generate new solutions to the bulk field equations linearised about the RN-AdS background. To see how this is done, let us begin with the simplest example of a complex scalar field with charge $q$ probing the RN-AdS black brane. In the Fourier domain, we can expand a field configuration in terms of planar waves:

$$
\int \frac{\mathrm{d} \omega \mathrm{d}^{d-1} \boldsymbol{k}}{(2 \pi)^{d}} \Phi(q, \omega, \zeta, \boldsymbol{k}) e^{-i \omega v+i \boldsymbol{k} \cdot \boldsymbol{x}} .
$$


Say this configuration solves the diffeomorphism/gauge covariant linear PDE in the bulk. How do we use the $\mathbb{Z}_{2}$ we constructed above to get new solutions? The quickest way to answer this is to use the KK (or the principal bundle) picture described above. To this end, we think of the above field as drawn from a KK decomposition of a higher dimensional scalar field of the form,

$$
\sum_{q \in \mathbb{Z}} e^{i q \varphi} \int \frac{\mathrm{d} \omega \mathrm{d}^{d-1} \boldsymbol{k}}{(2 \pi)^{d}} \Phi(q, \omega, \zeta, \boldsymbol{k}) e^{-i \omega v+i \boldsymbol{k} \cdot \boldsymbol{x}} .
$$

The diffeomorphism/gauge covariant linear PDE can then be lifted to a PDE covariant under higher dimensional diffeomorphisms. We perform a higher dimensional diffeomorphism $\{v \mapsto i \beta \zeta-v, \boldsymbol{x} \mapsto-\boldsymbol{x}, \varphi \mapsto \Lambda-\varphi\}$ on the Fourier domain expression given above. After relabelling the sum/integrals, we obtain,

$$
\sum_{q \in \mathbb{Z}} e^{i q \varphi} \int \frac{\mathrm{d} \omega \mathrm{d}^{d-1} \boldsymbol{k}}{(2 \pi)^{d}} e^{-\beta \omega \zeta-i q \wedge} \Phi(-q,-\omega, \zeta,-\boldsymbol{k}) e^{-i \omega v+i \boldsymbol{k} \cdot \boldsymbol{x}}
$$

The higher dimensional diffeomorphism covariance of the linear PDE then guarantees that the map

$$
\Phi(q, \omega, \zeta, \boldsymbol{k}) \longmapsto e^{-\beta \omega \zeta-i q \wedge} \Phi(-q,-\omega, \zeta,-\boldsymbol{k})
$$

generates new solutions. In fact, the pre-factor in the exponent shows that this map takes ingoing solutions that are analytic in $r$ to outgoing solutions which exhibit branch cuts. We can thus generate the Hawking modes from quasi-normal modes via the map,

$$
\Phi^{\text {in }}(q, \omega, \zeta, \boldsymbol{k}) \longmapsto e^{-\beta \omega \zeta-i q \wedge} \Phi^{\text {in }}(-q,-\omega, \zeta,-\boldsymbol{k}) \equiv \Phi^{\text {out }}(q, \omega, \zeta, \boldsymbol{k}) .
$$

The extension to other fields is straightforward. As a pertinent example, let us consider how this works for charged Dirac spinors. The main novelty in this case is the additional action of LLTs on spinor indices. The complex boost given in (2.10) acts on spinor indices via

$$
\mathfrak{T} \equiv \Gamma^{(\zeta)} \cdot \exp \left(\frac{\vartheta}{2} \Gamma^{(\zeta)} \Gamma^{(v)}\right)=\sqrt{f} \llbracket+\frac{1}{\sqrt{f}} \mathbb{\varpi}^{\dagger},
$$

where we have defined,

$$
\llbracket \equiv \frac{1}{2}\left(\Gamma^{(v)}+\Gamma^{(\zeta)}\right) .
$$

The Gamma matrix notation here is standard and our convention for the Clifford algebra is $\left\{\Gamma^{a}, \Gamma^{b}\right\}=2 \eta^{a b} \mathbb{1}$, with a mostly positive signature $\eta^{a b}$. The map from ingoing to outgoing solutions, in this case, is given by,

$$
\Psi^{\text {in }}(q, \omega, \zeta, \boldsymbol{k}) \longmapsto e^{-\beta \omega \zeta-i q \wedge} \mathfrak{T} \cdot \Psi^{\mathrm{in}}(-q,-\omega, \zeta,-\boldsymbol{k}) \equiv \Psi^{\mathrm{out}}(q, \omega, \zeta, \boldsymbol{k}) .
$$

In order to streamline the analysis that is to follow, we end this section with a discussion of how the quantities that we have introduced behave as they traverse the horizon cap. As noted before, the mock tortoise coordinate has a unit jump when it encircles $r=r_{h}$. 
Noting that the gauge field $\mathcal{A}_{v}$ has the value $-\mu$ at the horizon, we see that the jump in $e^{-i q \wedge}$ is the fugacity of the boundary theory,

$$
\lim _{\zeta \rightarrow 0} e^{-i q \wedge}=1, \quad \lim _{\zeta \rightarrow 1} e^{-i q \wedge}=e^{q \beta \mu} \equiv z .
$$

The rapidity parameter $\vartheta$, on the other hand, has a jump of $2 \pi i$ when it encircles the outer horizon - this can be traced to the phase $e^{2 \pi i}$ that the function $f$ picks up around the same path. As a result, every spinor index receives an additional 'fermionic' minus sign as it crosses the horizon cap. For a field of spin $s$, one gains the statistical factor $(-1)^{2 s} e^{-\beta \omega+q \beta \mu}$ which is identical to the factor gained in Euclidean path integrals as we traverse the thermal circle. As we will argue below, this factor is crucial to how the RN-SK geometry gets the physics of Hawking radiation right.

\section{The Dirac equation}

We will now apply the preceding slightly abstract discussion to the explicit example of a Dirac equation in the RN-SK background. The action for a bulk Dirac spinor $\Psi$ of charge $q$ and mass $m$ living on the RN-SK geometry is given by,

$$
\mathbb{S}_{\Psi}=\oint \mathrm{d} \zeta \int \mathrm{d}^{d} x \sqrt{-g} i \bar{\Psi}\left(\Gamma^{M} \mathbb{D}_{M}-m\right) \Psi+\left.\int \mathrm{d}^{d} x i \bar{\Psi} \mathcal{P}_{-}^{\zeta} \Psi\right|_{\zeta=0} ^{\zeta=1} .
$$

Let us explain each of the above terms. The first is the standard bulk Dirac action, that fixes the equations of motion to be the Dirac equation in this curved spacetime. We have introduced above the covariant Dirac derivative,

$$
\mathbb{D}_{M} \equiv \partial_{M}-i q \mathcal{A}_{M}+\frac{1}{4} \omega_{a b M} \Gamma^{a} \Gamma^{b}
$$

where $\mathcal{A}_{M}$ is the background gauge potential and $\omega_{a b M}$ are the spin-connection 1-forms derived from eq. (2.3).

The second term is a variational boundary term, added in to ensure that this action admits a well-posed variational principle when the spinors satisfy semi-Dirichlet boundary conditions [39-42] (more will be said about this below). When the above action is evaluated on-shell, the bulk term vanishes and only the boundary term contributes to the final answer. Notice that the boundary term receives contributions from both the left and right boundaries of the RN-SK saddle [2]. The operator $\mathcal{P}_{-}^{\zeta}$ involved in the definition of this term is one of two projection operators, defined as,

$$
\mathcal{P}_{ \pm}^{\zeta} \equiv \frac{1}{2}\left(\mathbb{1} \pm \Gamma^{(\zeta)}\right)
$$

The Dirac equation $\left(\Gamma^{M} \mathbb{D}_{M}-m\right) \Psi=0$, written out in the Fourier domain, takes the following form:

$$
\left\{\llbracket\left(\partial_{\zeta}+\beta \omega+\beta q \mathcal{A}_{v}+\frac{1}{2} \partial_{\zeta} \ln f\right)+\mathbb{\square}^{\dagger} f^{-1} \partial_{\zeta}-\frac{\beta}{2}\left(\Gamma^{(i)} k_{i}+i m r\right)\right\}\left(r^{\frac{d}{2}} \Psi\right)=0 .
$$


It can be explicitly checked that if $\Psi(q, \omega, \zeta, \boldsymbol{k})$ solves this equation, applying the transformation described in (2.20) to it generates another solution of the same equation.

The relevant AdS/CFT boundary conditions are,

$$
\lim _{\zeta \rightarrow 0} r^{\frac{d}{2}-m} \mathcal{P}_{+}^{\zeta} \Psi=\mathcal{P}_{+}^{\zeta} S_{0} \psi_{L}, \quad \lim _{\zeta \rightarrow 1} r^{\frac{d}{2}-m} \mathcal{P}_{+}^{\zeta} \Psi=\mathcal{P}_{+}^{\zeta} S_{0} \psi_{R}
$$

Here $S_{0}$ is a constant boundary-to-bulk matrix [2]. It is defined in terms of the ingoing boundary-to-bulk propagator $S^{\text {in }}(\omega, \zeta, \boldsymbol{k})$ as,

$$
S_{0} \equiv \lim _{\zeta \rightarrow 0} r^{\frac{d}{2}-m} S^{\mathrm{in}}(\omega, \zeta, \boldsymbol{k})=\lim _{\zeta \rightarrow 1} r^{\frac{d}{2}-m} S^{\mathrm{in}}(\omega, \zeta, \boldsymbol{k})
$$

The above boundary conditions are a 'doubled' version of the standard semi-Dirichlet conditions in AdS/CFT. These boundary conditions uniquely determine the solution on the RN-SK geometry.

To see this, we begin with the most general combination of ingoing and outgoing solutions:

$$
\begin{aligned}
\Psi(q, \omega, \zeta, \boldsymbol{k}) & =-S^{\mathrm{in}}(q, \omega, \zeta, \boldsymbol{k}) \psi_{\bar{F}}(q, \omega, \boldsymbol{k})-S^{\mathrm{out}}(q, \omega, \zeta, \boldsymbol{k}) \psi_{\bar{P}}(q, \omega, \boldsymbol{k}) e^{\beta(\omega-q \mu)}, \\
& =-S^{\mathrm{in}}(q, \omega, \zeta, \boldsymbol{k}) \psi_{\bar{F}}(q, \omega, \boldsymbol{k})-S^{\mathrm{rev}}(q, \omega, \zeta, \boldsymbol{k}) \psi_{\bar{P}}(q, \omega, \boldsymbol{k}) e^{\beta \omega(1-\zeta)+i q(i \beta \mu-\wedge)} .
\end{aligned}
$$

Here, we have defined,

$$
S^{\mathrm{rev}}(q, \omega, \zeta, \boldsymbol{k}) \equiv S^{\mathrm{in}}(-q,-\omega, \zeta,-\boldsymbol{k})
$$

and we have used the $\mathbb{Z}_{2}$ action detailed in the last section to get the outgoing Hawking solution.

The boundary conditions described above fix

$$
\psi_{\bar{F}} \equiv n_{\mathrm{FD}}\left(\psi_{R}-\psi_{L}\right)-\psi_{R}, \quad \psi_{\bar{P}} \equiv n_{\mathrm{FD}}\left(\psi_{R}-\psi_{L}\right)
$$

with

$$
n_{\mathrm{FD}} \equiv \frac{1}{e^{\beta(\omega-q \mu)}+1}
$$

being the familiar Fermi-Dirac factor at finite chemical potential. We recognise here the retarded-advanced basis (RA) for the boundary spinor sources [19, 43]. Thus, when written in the RA basis, the two combinations of sources precisely multiply the ingoing/quasinormal bulk-to-boundary propagator and the outgoing bulk-to-boundary propagator respectively. This is analogous to the corresponding statements at zero chemical potential $[2-4,7]$.

As pointed out in [2], the above fact can be used to argue why the generating function of correlations computed using our holographic prescription satisfy both the Schwinger-Keldysh collapse rules as well as the KMS conditions, i.e., the generating function cannot contain terms with only $\psi_{\bar{P}}$ or terms with only $\psi_{\bar{F}}$. Exactly the same argument is valid for the case of finite chemical potential presented here. We direct the reader to [2] for more details. 
Finally, in the Keldysh-rotated or average-difference basis, one gets,

$$
\Psi(v, \zeta, \boldsymbol{k})=S^{\mathrm{in}} \psi_{a}-\left\{\left(n_{\mathrm{FD}}-\frac{1}{2}\right) S^{\mathrm{in}}+n_{\mathrm{FD}} e^{\beta \omega(1-\zeta)+i q(i \beta \mu-\Lambda)} \mathfrak{T} \cdot S^{\mathrm{rev}}\right\} \psi_{d} .
$$

This basis makes the Schwinger-Keldysh collapse rules manifest, i.e., there are no terms in the generating function for boundary correlators that have only $\psi_{a}$ terms. Equivalently, correlation functions composed of only the difference operator $\mathcal{O}_{d}$ vanish. As we will see in section 4, the influence phase that we derive for the open EFT precisely has this property.

\section{Gradient expansion}

In this section, we will find the solution to the Dirac equation in gradient expansion, generalizing the work of [2] to charged black branes. We only consider the case of a massless spinor field for simplicity.

We begin by writing the most general solution $\Psi \equiv \Psi(v, \zeta, \boldsymbol{x})$ in a gradient expansion, compatible with rotational invariance as,

$$
\Psi=\frac{1}{r^{d / 2}}\left\{\mathbb{1}+C_{a}^{(1)} M^{a} \partial_{v}+D_{a}^{(1)} M^{a} \Gamma^{(i)} \partial_{i}+C_{a}^{(2)} M^{a} \partial_{v}^{2}+D_{a}^{(2)} M^{a} \partial_{i}^{2}+\ldots\right\} S_{0} \psi
$$

where $\psi \equiv \psi(v, \boldsymbol{x})$ is the boundary spinor acting as a source at the boundary. The functions $\left\{C^{(\mathrm{i})}, D^{(\mathrm{j})}, \ldots\right\}$ are some unknown functions of the radial direction $\zeta$. The matrices $M^{a}$ that appear in the above equation belong to the set $\left\{\mathbb{1}, \Gamma^{(\zeta)}\right\}$ and in order to satisfy the Dirac equation (3.4) at zero derivative order, we will fix $S_{0}$ to be a constant matrix annihilated

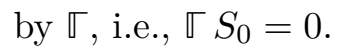

The bulk-to-boundary Green's function satisfying ingoing boundary conditions can be computed by substituting the above ansatz into the Dirac equation and solving it order by order in derivative expansion. In the Fourier domain we get an expression of the form,

$$
\begin{aligned}
S^{\text {in }}=\frac{1}{r^{d / 2}}\{ & +\frac{\beta}{2}\left(H \Gamma^{(\zeta)}-H_{(0)}\right) \Gamma^{(i)} k_{i}-\frac{\beta^{2} \omega}{2}\left(\widetilde{H} \Gamma^{(\zeta)}-\widetilde{H}_{(0)}\right) \Gamma^{(i)} k_{i} \\
& \left.\quad-\frac{\beta^{2} \boldsymbol{k}^{2}}{8}\left(f G^{2}-f_{(0)} G_{(0)}^{2}\right)-\frac{\beta^{2} \boldsymbol{k}^{2} H_{c}}{4}\left(H \Gamma^{(\zeta)}-H_{(0)}\right)+\ldots\right\} S_{0} .
\end{aligned}
$$

where $\boldsymbol{k}^{2}=k_{i} k^{i}$ and the subscript '(0)' denotes the value of the functions at the conformal boundary, $\zeta=0$. The unknown functions $H, \widetilde{H}$, and $G$ must satisfy the following radial differential equations:

$$
\frac{d}{d \zeta}\left(e^{i q \wedge} \sqrt{f} H\right)=e^{i q \wedge} \sqrt{f}, \quad \frac{d}{d \zeta}\left(e^{i q \wedge} \sqrt{f} \widetilde{H}\right)=e^{i q \wedge} \sqrt{f} H, \quad \frac{d}{d \zeta}(\sqrt{f} G)=\sqrt{f} .
$$

Since we want an ingoing solution, we take $H, \widetilde{H}$, and $G$ to be analytic at the outer horizon which then implies that the functions $\sqrt{f} H, \sqrt{f} \widetilde{H}$ and $\sqrt{f} G$ should vanish at the outer horizon. With this boundary condition specified, the above ODEs have a unique solution. 
Given the above ingoing solution, the corresponding solution on the RN-SK geometry follows by using the formulae derived in section 3 . We will end this section by quoting the result for the influence phase in average-difference basis:

$$
\mathbb{S}_{\mathrm{IF}}\left[\psi_{a}, \psi_{d}\right]=\left.\int \mathrm{d}^{d} x r^{d} i \bar{\Psi} \mathcal{P}_{-}^{\zeta} \Psi\right|_{\zeta=0} ^{\zeta=1}=\int \mathrm{d}^{d} x i\left\{\bar{\psi}_{a} \mathcal{S}^{d a} \psi_{d}+\bar{\psi}_{d} \mathcal{S}^{a d} \psi_{a}+\bar{\psi}_{d} \mathcal{S}^{a a} \psi_{d}\right\}
$$

where $\mathcal{S}^{a d}, \mathcal{S}^{d a}$, and $\mathcal{S}^{a a}$ are the retarded, advanced and Keldysh Green's functions respectively. When the dimension of the boundary theory, $d$, is odd, their explicit forms are given by,

$$
\begin{aligned}
\mathcal{S}^{a d}= & \gamma^{(v)}-\beta H_{(0)} \gamma^{(i)} k_{i}+\beta^{2} \omega \gamma^{(i)} k_{i} \widetilde{H}_{(0)}+\frac{\beta^{2} \boldsymbol{k}^{2}\left(H_{(0)}\right)^{2}}{2} \gamma^{(v)}+\ldots \\
\mathcal{S}^{d a}= & -\left(\gamma^{(v)}+\beta H_{(0)}^{\star} \gamma^{(i)} k_{i}+\beta^{2} \omega \gamma^{(i)} k_{i} \widetilde{H}_{(0)}^{\star}+\frac{\beta^{2} \boldsymbol{k}^{2}\left(H_{(0)}^{\star}\right)^{2}}{2} \gamma^{(v)}+\ldots\right) \\
\mathcal{S}^{a a}= & \mathfrak{e} \gamma^{(v)}+\frac{\beta \omega\left(1-\mathfrak{e}^{2}\right)}{2} \gamma^{(v)}-\frac{\beta^{2} \omega^{2}\left[\mathfrak{e}\left(1-\mathfrak{e}^{2}\right)\right]}{4} \gamma^{(v)}+\frac{\mathfrak{e} \beta\left(H_{(0)}^{\star}-H_{(0)}\right)}{2} \gamma^{(i)} k_{i} \\
& +\frac{\beta^{2} \omega}{4} \gamma^{(i)} k_{i}\left(\left(1-\mathfrak{e}^{2}\right)\left(H_{(0)}^{\star}-H_{(0)}\right)+2 \mathfrak{e}\left(\widetilde{H}_{(0)}^{\star}+\widetilde{H}_{(0)}\right)\right) \\
& +\frac{\mathfrak{e} \beta^{2} \boldsymbol{k}^{2}\left[\left(H_{(0)}^{\star}\right)^{2}+H_{(0)}^{2}\right]}{4} \gamma^{(v)}+\ldots
\end{aligned}
$$

where we have expanded the Fermi-Dirac factors in powers of $\omega$ and the superscript ' $\star$ ' denotes the replacement $q \rightarrow-q$ in the arguments of the functions. We use the letter $\mathfrak{e}$ to denote the ground state charge

$$
\mathfrak{e} \equiv \frac{1}{1+e^{-q \beta \mu}}-\frac{1}{1+e^{q \beta \mu}},
$$

which is the difference between the ground-state occupation of fermionic particles and antiparticles. A similar set of expressions can be obtained $d$ is even, as described in [2]. This influence phase obeys the 2-point KMS relation which takes the following form in the a-d basis,

$$
\mathcal{S}^{a a}=\frac{1}{2} \frac{e^{\beta \omega}-z}{e^{\beta \omega}+z}\left(\mathcal{S}^{a d}-\mathcal{S}^{d a}\right) .
$$

In the limit $\omega, \boldsymbol{k} \rightarrow 0$, the influence phase has a very simple form,

$$
\mathrm{S}_{\mathrm{IF}}=\int \mathrm{d}^{d} x i\left(\psi_{a}^{\dagger} \psi_{d}-\psi_{d}^{\dagger} \psi_{a}-\mathfrak{e} \psi_{d}^{\dagger} \psi_{d}\right)
$$

Finally, in the limit of uncharged black branes or vanishing chemical potential $(q, \mu \rightarrow 0)$, all these Green's functions and the influence phase match with the results derived in [2].

\section{Conclusions and future directions}

In this note we extended the prescription for gravitational Schwinger-Keldysh saddles to the case of charged black branes. This geometry is dual to the real-time evolution of a CFT 
held at a finite temperature and chemical potential. Our prescription is a straightforward generalisation of the original grSK prescription [1-4]. In this geometry, we describe how to obtain the outgoing or Hawking modes from the ingoing quasi-normal modes via a CPT transformation.

In order to test the above prescription, we probe the RN-AdS black brane with Dirac fermions charged under the gauge field. Using this solution, we derive the influence phase of a probe fermion interacting with the holographic CFT [2]. We show that the correlators derived using this holographic prescription satisfy the desired KMS relations dressed with the correct Fermi-Dirac factors. This is our central result which generalises the results of [2] to include the chemical potential of the CFT. Further, specialising to the case of massless fields, we derive the quasi-normal modes up to second order in a boundary derivative expansion.

A natural follow up to this study would be to examine the near-extremal limit. In this limit, the outer and the inner horizons come close to each other and can lead to the pinching of the holographic radial contour. It would be interesting to see how this should be dealt with and make contact with the derivative expansion in near-extremal branes recently claimed in [44].

\section{Acknowledgments}

It is with great pleasure that we thank Bidisha Chakraborty, Greg Henderson, Chris Herzog, Aswin P. M., Suvrat Raju, Mukund Rangamani, Omkar Shetye and Spenta Wadia for useful discussions. KR is supported by the Rhodes Trust via a Rhodes Scholarship. The authors would like to acknowledge their debt to the people of India for their sustained and generous support to research in the basic sciences.

Open Access. This article is distributed under the terms of the Creative Commons Attribution License (CC-BY 4.0), which permits any use, distribution and reproduction in any medium, provided the original author(s) and source are credited.

\section{References}

[1] P. Glorioso, M. Crossley and H. Liu, A prescription for holographic Schwinger-Keldysh contour in non-equilibrium systems, arXiv:1812.08785 [INSPIRE].

[2] R. Loganayagam, K. Ray and A. Sivakumar, Fermionic open EFT from holography, arXiv:2011.07039 [INSPIRE].

[3] B. Chakrabarty, J. Chakravarty, S. Chaudhuri, C. Jana, R. Loganayagam and A. Sivakumar, Nonlinear Langevin dynamics via holography, JHEP 01 (2020) 165 [arXiv:1906.07762] [INSPIRE].

[4] C. Jana, R. Loganayagam and M. Rangamani, Open quantum systems and Schwinger-Keldysh holograms, JHEP 07 (2020) 242 [arXiv: 2004.02888] [INSPIRE].

[5] D.T. Son and A.O. Starinets, Minkowski space correlators in AdS/CFT correspondence: recipe and applications, JHEP 09 (2002) 042 [hep-th/0205051] [INSPIRE].

[6] C.P. Herzog and D.T. Son, Schwinger-Keldysh propagators from AdS/CFT correspondence, JHEP 03 (2003) 046 [hep-th/0212072] [INSPIRE]. 
[7] D.T. Son and D. Teaney, Thermal noise and stochastic strings in AdS/CFT, JHEP 07 (2009) 021 [arXiv:0901.2338] [INSPIRE].

[8] K. Skenderis and B.C. van Rees, Real-time gauge/gravity duality, Phys. Rev. Lett. 101 (2008) 081601 [arXiv:0805.0150] [INSPIRE].

[9] K. Skenderis and B.C. van Rees, Real-time gauge/gravity duality: prescription, renormalization and examples, JHEP 05 (2009) 085 [arXiv:0812.2909] [INSPIRE].

[10] B.C. van Rees, Real-time gauge/gravity duality and ingoing boundary conditions, Nucl. Phys. B Proc. Suppl. 192-193 (2009) 193 [arXiv:0902 .4010] [INSPIRE].

[11] R.G. Leigh and N. Nguyen hoang, Real-time correlators and non-relativistic holography, JHEP 11 (2009) 010 [arXiv: 0904.4270] [INSPIRE].

[12] G.C. Giecold, Fermionic Schwinger-Keldysh propagators from AdS/CFT, JHEP 10 (2009) 057 [arXiv: 0904 . 4869] [INSPIRE].

[13] E. Barnes, D. Vaman and C. Wu, Holographic real-time non-relativistic correlators at zero and finite temperature, Phys. Rev. D 82 (2010) 125042 [arXiv:1007.1644] [INSPIRE].

[14] E. Barnes, D. Vaman, C. Wu and P. Arnold, Real-time finite-temperature correlators from AdS/CFT, Phys. Rev. D 82 (2010) 025019 [arXiv: 1004.1179] [INSPIRE].

[15] M. Botta-Cantcheff, P.J. Martínez and G.A. Silva, Interacting fields in real-time AdS/CFT, JHEP 03 (2017) 148 [arXiv:1703.02384] [INSPIRE].

[16] J. de Boer, M.P. Heller and N. Pinzani-Fokeeva, Holographic Schwinger-Keldysh effective field theories, JHEP 05 (2019) 188 [arXiv:1812.06093] [INSPIRE].

[17] J.S. Schwinger, Brownian motion of a quantum oscillator, J. Math. Phys. 2 (1961) 407 [INSPIRE].

[18] L.V. Keldysh, Diagram technique for nonequilibrium processes, Zh. Eksp. Teor. Fiz. 47 (1964) 1515 [Sov. Phys. JETP 20 (1965) 1018] [INSPIRE].

[19] K.-C. Chou, Z.-B. Su, B.-L. Hao and L. Yu, Equilibrium and nonequilibrium formalisms made unified, Phys. Rept. 118 (1985) 1 [INSPIRE].

[20] A. Kamenev, Field theory of non-equilibrium systems, Cambridge University Press, Cambridge, U.K. (2011).

[21] M.L. Bellac, Thermal field theory, Cambridge University Press, Cambridge, U.K. (2011).

[22] J. Rammer, Quantum field theory of non-equilibrium states, Cambridge University Press, Cambridge, U.K. (2007).

[23] N.P. Landsman and C.G. van Weert, Real and imaginary time field theory at finite temperature and density, Phys. Rept. 145 (1987) 141 [INSPIRE].

[24] N. Iqbal, H. Liu and M. Mezei, Lectures on holographic non-Fermi liquids and quantum phase transitions, in Theoretical Advanced Study Institute in Elementary Particle Physics. String theory and its applications: from meV to the Planck scale, World Scientific, Singapore (2011), pg. 707 [arXiv:1110.3814] [INSPIRE].

[25] S.A. Hartnoll, A. Lucas and S. Sachdev, Holographic quantum matter, arXiv:1612.07324 [INSPIRE].

[26] S.A. Hartnoll, Lectures on holographic methods for condensed matter physics, Class. Quant. Grav. 26 (2009) 224002 [arXiv:0903.3246] [INSPIRE]. 
[27] J. McGreevy, Holographic duality with a view toward many-body physics, Adv. High Energy Phys. 2010 (2010) 723105 [arXiv:0909.0518] [INSPIRE].

[28] C.P. Herzog, Lectures on holographic superfluidity and superconductivity, J. Phys. A 42 (2009) 343001 [arXiv:0904.1975] [inSPIRE].

[29] B. Doucot, C. Ecker, A. Mukhopadhyay and G. Policastro, Density response and collective modes of semiholographic non-Fermi liquids, Phys. Rev. D 96 (2017) 106011 [arXiv: 1706.04975] [INSPIRE].

[30] S.-S. Lee, Low energy effective theory of Fermi surface coupled with U(1) gauge field in $2+1$ dimensions, Phys. Rev. B 80 (2009) 165102 [arXiv:0905.4532] [INSPIRE].

[31] D.F. Mross, J. McGreevy, H. Liu and T. Senthil, A controlled expansion for certain non-Fermi liquid metals, Phys. Rev. B 82 (2010) 045121 [arXiv: 1003.0894] [INSPIRE].

[32] S.A. Hartnoll, P.K. Kovtun, M. Muller and S. Sachdev, Theory of the Nernst effect near quantum phase transitions in condensed matter, and in dyonic black holes, Phys. Rev. B 76 (2007) 144502 [arXiv:0706.3215] [INSPIRE].

[33] J. Zaanen, Y. Liu, Y.-W. Sun and K. Schalm, Holographic duality in condensed matter physics, Cambridge University Press, Cambridge, U.K. (2015).

[34] R. Kubo, Statistical mechanical theory of irreversible processes. 1. General theory and simple applications in magnetic and conduction problems, J. Phys. Soc. Jap. 12 (1957) 570 [INSPIRE].

[35] P.C. Martin and J.S. Schwinger, Theory of many particle systems. 1, Phys. Rev. 115 (1959) 1342 [INSPIRE].

[36] M. Rangamani, Gravity and hydrodynamics: lectures on the fluid-gravity correspondence, Class. Quant. Grav. 26 (2009) 224003 [arXiv:0905.4352] [INSPIRE].

[37] V.E. Hubeny, S. Minwalla and M. Rangamani, The fluid/gravity correspondence, in Theoretical Advanced Study Institute in Elementary Particle Physics. String theory and its applications: from meV to the Planck scale, (2012), pg. 348 [arXiv:1107.5780] [INSPIRE].

[38] N. Ceplak, K. Ramdial and D. Vegh, Fermionic pole-skipping in holography, JHEP 07 (2020) 203 [arXiv: 1910.02975] [INSPIRE].

[39] M. Henningson and K. Sfetsos, Spinors and the AdS/CFT correspondence, Phys. Lett. B 431 (1998) 63 [hep-th/9803251] [INSPIRE].

[40] W. Mueck and K.S. Viswanathan, Conformal field theory correlators from classical field theory on anti-de Sitter space. 2. Vector and spinor fields, Phys. Rev. D 58 (1998) 106006 [hep-th/9805145] [INSPIRE].

[41] M. Henneaux, Boundary terms in the AdS/CFT correspondence for spinor fields, in International meeting on mathematical methods in modern theoretical physics (ISPM 98), (1998), pg. 161 [hep-th/9902137] [INSPIRE].

[42] N. Iqbal and H. Liu, Real-time response in AdS/CFT with application to spinors, Fortsch. Phys. 57 (2009) 367 [arXiv:0903.2596] [InSPIRE].

[43] S. Chaudhuri, C. Chowdhury and R. Loganayagam, Spectral representation of thermal OTO correlators, JHEP 02 (2019) 018 [arXiv:1810.03118] [INSPIRE].

[44] U. Moitra, S.K. Sake and S.P. Trivedi, Near-extremal fluid mechanics, JHEP 02 (2021) 021 [arXiv: 2005.00016] [INSPIRE]. 